\title{
Acute unilateral submandibular gland swelling associated with the laryngeal mask airway
}

Thangavelautham Suhitharan ${ }^{1}$, MBBS, Sathyendran Seevanayagam ${ }^{1,2}$, MBBS, FANZCA, Francis Christopher $\underline{\text { Parker }}^{3}$, MBBS, FANZCA, Wendy Hui Ling Teoh ${ }^{4}$, MBBS, FANZCA

\begin{abstract}
We describe a rare complication of acute unilateral submandibular gland swelling following the use of laryngeal mask airway (LMA) in two patients with otherwise uneventful perioperative airway management. This is likely to be a consequence of the pressure exerted by the airway cuff on the tissues within the submandibular triangle. As this complication is rarely reported, its true incidence may in fact be higher, suggesting a need for greater attention on LMA cuff pressures and degree of cuff inflation. We discuss the presenting clinical features, pathophysiology and utilisation of ultrasonographic confirmation of sialadenopathy, and review the current anaesthetic literature to raise awareness of this unusual and under-reported complication of LMA. This complication can be mitigated by incorporating routine manometric checks and limiting intracuff pressures to $<60 \mathrm{cmH}_{2} \mathrm{O}$, potentially avoiding LMA insertions in patients with sialolithiasis and avoiding the use of nitrous oxide.
\end{abstract}

Keywords: laryngeal mask airway, salivary gland, sialadenopathy, submandibular gland swelling, ultrasonography

\section{INTRODUCTION}

The laryngeal mask airway (LMA) has revolutionised airway management since its introduction 30 years ago. The prevalent use of such second-generation supraglottic airways with a gastric drain tube has resulted in the replacement of the tracheal tube in a greater variety of clinical scenarios, such as laparoscopic surgeries, in which endotracheal intubation was once deemed to be the traditional stronghold of securing the airway. ${ }^{(1)}$ Fortunately, the prolific use of LMA has not been paralleled by emerging reports of increased incidences of LMA-related complications. Nonetheless, given the prevalence and popularity of LMA, hitherto published reports of LMArelated tissue complications include unilateral hypoglossal nerve paralysis, ${ }^{(2)}$ lingual and inferior alveolar nerve injury, ${ }^{(3,4)}$ and rarely, submandibular gland swelling. ${ }^{(5)}$ Not all postoperative swellings are benign. Pharyngeal packs have been known to lead to massive tongue swelling, ${ }^{(6)}$ and the occurrence of 'anaesthesia mumps' (acute postoperative sialadenitis) has also been reported after general anaesthesia, necessitating emergent intubation for airway compromise. ${ }^{(7)}$ We herein describe two cases of LMA-related unilateral submandibular gland swelling, which were confirmed using bedside ultrasonography.

\section{CASE REPORT}

\section{Case 1}

A healthy, 29-year-old Caucasian woman weighing $69 \mathrm{~kg}$ presented for arthroscopic surgery of her right shoulder. We administered $1.5 \mathrm{mg}$ of intravenous (IV) midazolam, and a right interscalene block, using $20 \mathrm{~mL}$ of $0.75 \%$ ropivacaine, was established with a nerve stimulator. Anaesthesia was induced using propofol, and a size-4 classic LMA (with the cuff fully deflated) was inserted smoothly at the first attempt. Subsequently, $20 \mathrm{~mL}$ of air was introduced into the cuff, with no air leakage during manual ventilation. Anaesthesia was maintained using sevoflurane in a $40 \%$ oxygen $\left(\mathrm{O}_{2}\right)$ to $60 \%$ nitrous oxide $\left(\mathrm{N}_{2} \mathrm{O}\right)$ mixture. The patient was put in the beach chair position for surgery and covered with sterile drapes. The surgery lasted 75 mins, with the LMA and patient's head secured in neutral position. Upon removal of the surgical drapes, a large right-sided submandibular mass was noted (Fig. 1a). The mass was soft, round, non-tender and non-pulsatile, with a diameter of $7-8 \mathrm{~cm}$ and no surface discolouration. The patient was neither stridorous nor dyspnoeic before and after the removal of LMA. The initial differential diagnosis of the mass was haematoma secondary to interscalene block. The patient was transferred to the recovery area for monitoring. Bedside ultrasonography using a Hitachi Eub-8500 ultrasonography machine and linear probe revealed an abnormally enlarged right submandibular gland (Fig. 1b) compared to the left (Fig. 1c). However, this spontaneously and completely subsided within 20 mins.

\section{Case 2}

A healthy, 40-year-old Chinese man weighing $71 \mathrm{~kg}$ had a left forearm laceration repaired under general anaesthesia, during which a size-4 LMA Supreme ${ }^{\mathrm{TM}}$ was inserted. He was premedicated with $2 \mathrm{mg}$ of IV midazolam and $5 \mathrm{mg}$ of IV morphine. Anaesthesia was induced using propofol. LMA was inserted smoothly, and its cuff was inflated with $20 \mathrm{~mL}$ of air.

\footnotetext{
${ }^{1}$ Department of Anaesthesia and Surgical Intensive Care, Changi General Hospital, Singapore, ${ }^{2}$ Department of Anaesthesia, Northern Hospital, Epping, ${ }^{3}$ Department of Anaesthesia, Austin Health, Heidelberg, Victoria, Australia, ${ }^{4}$ Department of Women's Anaesthesia, KK Women's and Children's Hospital, Singapore

Correspondence: Dr Suhitharan Thangavelautham, Anaesthesiology Resident, Department of Anaesthesia and Surgical Intensive Care, Changi General Hospital, 2 Simei Street 3, Singapore 529889. tsuhitharan@yahoo.com
} 

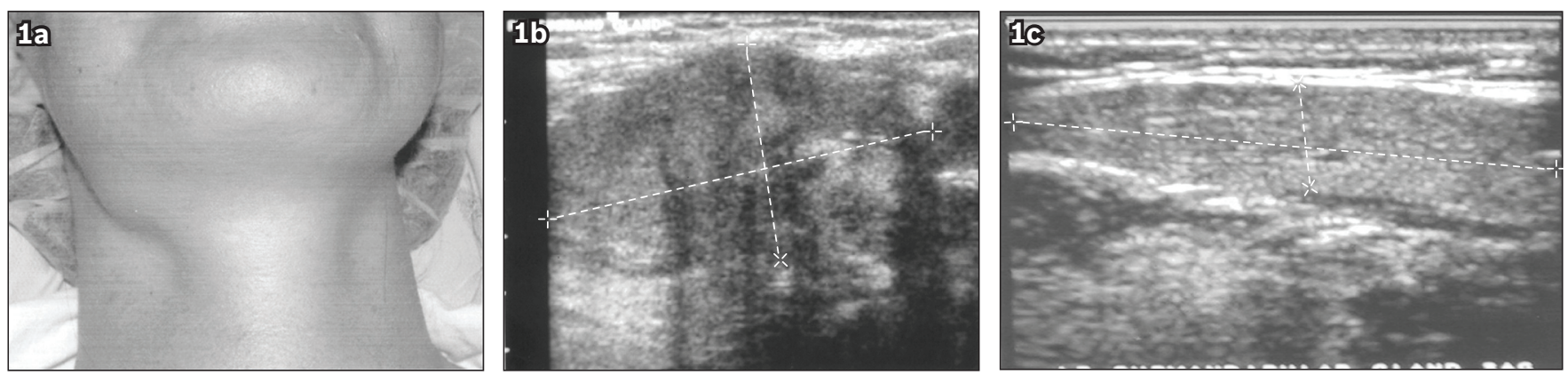

Fig. 1 Case 1: (a) Photograph shows the right submandibular mass after removal of LMA; (b) Ultrasonographic image shows an abnormally enlarged right submandibular gland measuring $6.6 \mathrm{~cm} \times 3.6 \mathrm{~cm}$ (refer to calliper markings); (c) Ultrasonographic image shows a normal-looking left submandibular gland in the sagittal plane.
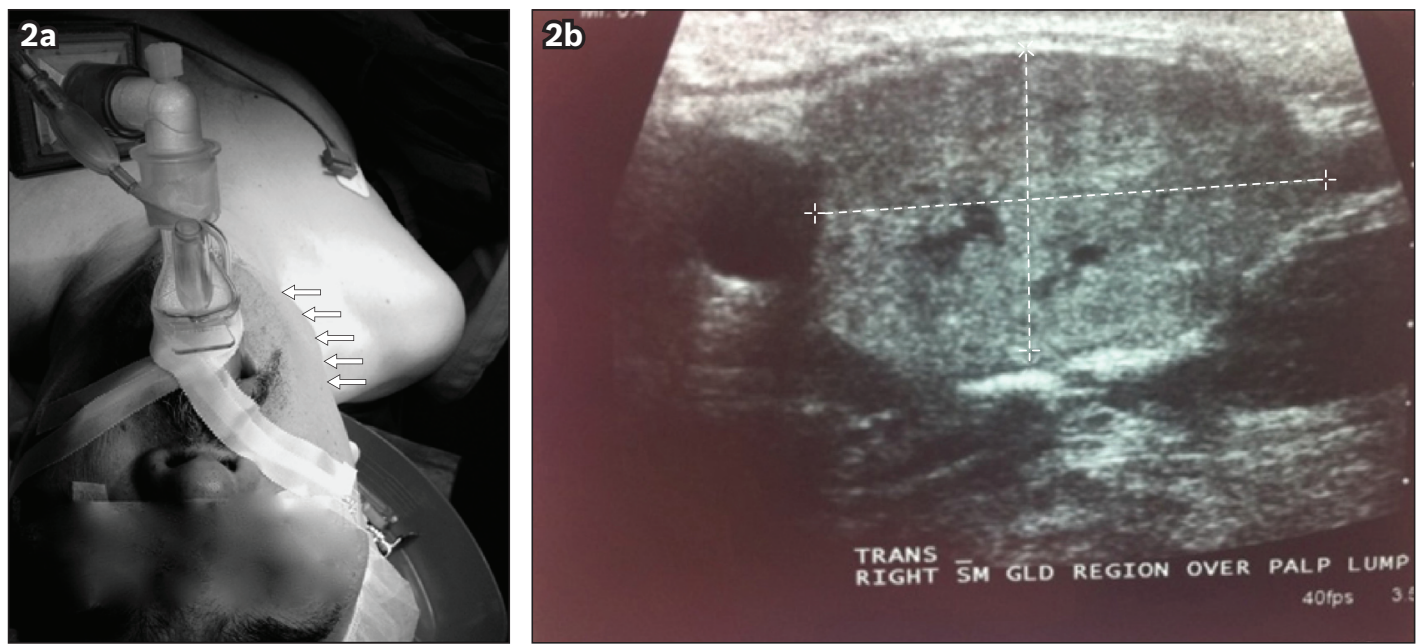

Fig. 2 Case 2: (a) Perioperative photograph taken at the end of surgery shows a right submandibular mass (arrows) (b) Ultrasonography image shows an abnormally enlarged right submandibular gland measuring $4 \mathrm{~cm} \times 3 \mathrm{~cm}($ refer to calliper markings).

Anaesthesia was maintained with sevoflurane in a $40 \% \mathrm{O}_{2}$ to $60 \% \mathrm{~N}_{2} \mathrm{O}$ mixture throughout the 95-min surgery, which involved neurovascular injury repair. At the completion of the surgery, the patient was noted to have a soft, rounded swelling in the right submandibular area (Fig. 2a), measuring approximately $5 \mathrm{~cm}$ in diameter, with no airway compromise. The swelling was non-pulsatile and of normal colour and temperature. Ultrasonographic images obtained 12 mins after LMA removal revealed swelling of the right submandibular gland, measuring $4 \mathrm{~cm} \times 3 \mathrm{~cm} \times 2 \mathrm{~cm}$ (Fig. 2b). This, however, resolved over the next 15 mins in recovery, without any sequelae. The patient was discharged back to the ward with reassurance.

\section{DISCUSSION}

Acute salivary gland swelling associated with anaesthesia is a rare phenomenon. It was first described in 1968 by Attas et $\mathrm{al}^{(8)}$ as an enlargement in the salivary glands with rubbery consistency, occurring soon after a smooth anaesthetic induction and intubation. The swelling was described to have gradually decreased in size, becoming barely palpable after 30 mins. ${ }^{(8)}$ Gupta described an acute transient unilateral submandibular swelling in an intubated patient 3 mins after insertion of a Guedel airway (its tip was thought to temporarily occlude the submandibular duct or base of the tongue by pushing the gland downwards). ${ }^{(9)}$ Hooda and Gupta in 1998 first reported the implication of LMA in submandibular salivary gland swelling. ${ }^{(5)}$

The submandibular glands are $U$-shaped salivary glands the size of a thumb. These glands lie along the body of the mandible in the anterior part of the submandibular triangle, and are bound by the digastric muscle and the inferior margin of the mandibular body, with the mylohyoid muscle forming the floor. Other contents of the submandibular triangle include the hyoglossus muscle, lingual and facial arteries, and lingual and hypoglossal nerves. The submandibular duct arises from the portion of the gland that lies between the mylohyoid and hyoglossus muscles. The duct passes deep and then superficial to the lingual nerve. It opens by one to three orifices on a small sublingual papilla beside the lingual frenulum on the floor of the mouth. The gland is supplied by parasympathetic, secretomotor fibres from the submandibular ganglion, and produces $70 \%$ of the salivary volume in humans. ${ }^{(10)}$

To the best of our knowledge, this LMA-associated complication that results in the swelling of the submandibular gland has only been reported twice in English language journals. ${ }^{(3,5)}$ Hence, awareness of this problem within the anaesthetic community is low. We believe that this complication 
is under-recognised, partly due to the subtlety of its presentation. Complications involving the submandibular glands of patients under general anaesthesia have also been attributed to other factors such as drugs (e.g. atropine, succinylcholine), overactive pharyngeal reflexes stimulating the submandibular gland via parasympathetic nerves, raised intrathoracic pressure and deformation of the submandibular glands/ducts due to the pressure exerted on the tissues of the submandibular triangle by an apparently clinically wellplaced LMA. ${ }^{(5,8,11)}$

In the present two cases, our patients were not exposed to the aforementioned drugs that are known to be commonly implicated with LMA-associated submandibular gland swelling, and there was no clinical evidence during the induction, maintenance or awakening phases of anaesthesia to suggest that raised intrathoracic pressure or hyperactive pharyngeal reflexes led to the complications. We thus suspect that the unilateral submandibular gland swelling noted in both our patients was due to deformation of the glandular tissue, which was caused by the pressure exerted by the LMA cuff on the submandibular triangle. Routine manometric check of intracuff pressures was not performed in these two cases. In the present two cases, although the LMA was inserted smoothly at the first attempt and secured in midline with no air leak on manual ventilation, with the same midline positions confirmed at the end of surgery, a malpositioned LMA capable of adequate function cannot be ruled out as a possible cause.

Ogata et al demonstrated ultrasonographically in ten patients with LMAs in situ that the degree of submandibular gland compression increased markedly as the pressure in the LMA cuff increased from $50 \mathrm{cmH}_{2} \mathrm{O}$ to $150 \mathrm{cmH}_{2} \mathrm{O}$; the width and length of the gland were significantly unchanged between $0 \mathrm{cmH}_{2} \mathrm{O}$ and $50 \mathrm{cmH}_{2} \mathrm{O}$. ${ }^{(11)}$ It has also been demonstrated that higher LMA intracuff pressures and cuff volumes result in higher transmitted pharyngeal mucosal pressure by the anterior base of the LMA cuff. ${ }^{(12)}$ Previous ultrasonographic findings also showed that the LMA easily pressed the pharynx into the submandibular triangle. ${ }^{(11)}$ Thus, in this way, the submandibular glands could easily become deformed without the musculoskeletal support of the mylohyoid muscle. This deformation is hypothesised to be more significant with an LMA than an endotracheal tube because the former occupies a greater volume of the oral cavity. In the clinical setting, LMA cuff pressures beyond $60 \mathrm{cmH}_{2} \mathrm{O}$ are likely to occur in the absence of routine manometric checks, hence there is a need for greater vigilance to exclude cuff-related complications. ${ }^{(13)}$

I-gel ${ }^{\mathrm{TM}}$ (Intersurgical Ltd, Wokingham, UK) is a unique, disposable supraglottic airway device with a soft, gel-like cuff (made of thermoplastic elastomer) and a gastric drain port that was introduced at the same time as the LMA Supreme ${ }^{\mathrm{TM}}$ in 2007. The non-inflatable cuff of the I-gel ${ }^{\mathrm{TM}}$ is designed to create a perilaryngeal anatomical seal, which was found in cadaveric studies to decrease the risk of compression trauma. ${ }^{(14)}$ Clinically, LMA Supreme ${ }^{\mathrm{TM}}$ and I-gel ${ }^{\mathrm{TM}}$ are similar in performance, with comparable oropharyngeal leak pressures of $26.4 \pm 5.1 \mathrm{cmH}_{2} \mathrm{O}$ and $25.0 \pm 5.7 \mathrm{cmH}_{2} \mathrm{O}$, respectively $(p=0.18) .{ }^{(1)}$ However, I-gel ${ }^{\mathrm{TM}}$ was found to result in lower incidences of postoperative sore throat, dysphagia and neck complaints, compared to the disposable laryngeal mask. ${ }^{(15)}$ This is due to the inability of the unique non-inflatable cuff (found in I-gel ${ }^{\mathrm{TM}}$ ) to reach high intracuff pressures, making it a valuable asset.

The use of $\mathrm{N}_{2} \mathrm{O}$ during the maintenance of anaesthesia may further exacerbate cuff volume and pressures.(16) Notwithstanding that, risk factors such as pre-existing submandibular gland pathology are also difficult to identify in the first place. The reported incidence of sialolithiasis is 1 per 10,000-20,000 people, of whom many may be asymptomatic. ${ }^{(17)}$ Thus, it would be prudent to avoid the use of LMA in anyone with a history of salivary colic pain or known stones, and accord judicious attention to the submandibular region. Salivary stones as small as $2 \mathrm{~mm} \times 3 \mathrm{~mm}$ can be recognised as high-level reverberation echoes on ultrasonography, accompanied by posterior acoustic shadows or hyperechoic masses; the detection rate of salivary stones using the ultrasonographic method is as high as that when using sialography. ${ }^{(18)}$ With the advent of portable ultrasonography machines, and the widening domain of anaesthetists as perioperative physicians, the use of simple and noninvasive bedside ultrasonographic examination to evaluate sialolithiasis could probably be performed more frequently, especially when there is acute infection of the gland.

We believe it is important for anaesthetists to be aware of this unusual and under-reported complication of LMA, not only because of its benign course that resolves spontaneously (thus requiring no expensive or invasive investigations), but also because it can largely be avoided by incorporating routine manometric checks, limiting intracuff pressures to $<60 \mathrm{cmH}_{2} \mathrm{O}$, avoiding factors such as the use of $\mathrm{N}_{2} \mathrm{O}$, and using LMAs with an inbuilt cuff pilot indicator for safety (e.g. GuardianCPV Laryngeal mask; Ultimate Medical, Australia). We encourage all anaesthetists to give attention to LMA intracuff pressure in an effort to collectively minimise the incidence of complications secondary to high cuff pressure.

\section{REFERENCES}

1. Teoh WH, Lee KM, Suhitharan T, et al. Comparison of the LMA Supreme vs the i-gel in paralysed patients undergoing gynaecological laparoscopic surgery with controlled ventilation. Anaesthesia 2010; 65:1173-9.

2. Nagai K, Sakuramoto C, Goto F. Unilateral hypoglossal nerve paralysis following the use of the laryngeal mask airway. Anaesthesia 1994; 49:603-4.

3. Brimacombe J, Keller C. Salivary gland swelling and lingual nerve injury with the ProSeal laryngeal mask airway. Eur J Anaesthesiol 2005; 22:954-5.

4. Hanumanthaiah D, Masud S, Ranganath A. Inferior alveolar nerve injury with laryngeal mask airway: a case report. J Med Case Rep 2011; 5:122.

5. Hooda S, Gupta SK. Acute transient sialadenopathy associated with laryngeal mask airway. Anesth Analg 1998; 87:1438-40.

6. Kawaguchi M, Sakamoto T, Ohnishi H, Karasawa J. Pharyngeal packs 
can cause massive swelling of the tongue after neurosurgical procedures. Anesthesiology 1995; 83:434-5.

7. Quinn ME, Quinn TD, Mian AZ, Sakai O. Imaging findings of "anesthesia mumps" (acute postoperative sialadenitis) after general anesthesia. J Comput Assist Tomogr 2012; 36:745-8.

8. Attas M, Sabawala PB, Keats AS. Acute transient sialadenopathy during induction of anesthesia. Anesthesiology 1968; 29:1050-2.

9. Gupta R. Unilateral transient sialadenopathy: another complication of oropharyngeal airway. Anesthesiology 1998; 88:551-2.

10. Moore KL. Salivary glands. In: Paul J Kelly, ed. Clinically Oriented Anatomy. Baltimore: Lippincott, Williams \&Wilkins, 1992; 948-9.

11. Ogata J, Minami K, Oishi M, Tamura H, Shigematsu A. The influence of the laryngeal mask airway on the shape of the submandibular gland. Anesth Analg 2001; 93:1069-72.

12. Brimacombe J, Keller C. A comparison of pharyngeal mucosal pressure and airway sealing pressure with the laryngeal mask airway in anesthetized adult patients. Anesth Analg 1998; 87:1379-82.
13. Seet E, Yousaf F, Gupta S, et al. Use of manometry for laryngeal mask airway reduces postoperative pharyngolaryngeal adverse events: a prospective, randomized trial. Anesthesiology 2010; 112:652-7.

14. Levitan RM, Kinkle WC. Initial anatomic investigations of the I-gel airway: a novel supraglottic airway without inflatable cuff. Anaesthesia 2005; 60:1022-6.

15. Keijzer C, Buitelaar DR, Efthymiou KM, et al. A comparison of postoperative throat and neck complaints after the use of the i-gel and the La Premiere disposable laryngeal mask: a double-blinded, randomized, controlled trial. Anesth Analg 2009; 109:1092-5.

16. Lumb AB, Wrigley MW. The effect of nitrous oxide on laryngeal mask cuff pressure. In vitro and in vivo studies. Anaesthesia 1992; 47:320-3.

17. Marchal F, Dulguerov P. Sialolithiasis management: the state of the art. Arch Otolaryngol Head Neck Surg 2003; 129:951-6.

18. Yoshimura Y, Inoue Y, Odagawa T. Sonographic examination of sialolithiasis. J Oral Maxillofac Surg 1989; 47:907-12. 